\title{
Ingenuity pathway analysis of human facet joint tissues: Insight into facet joint osteoarthritis
}

\author{
CHU CHEN ${ }^{*}$, SHENGYU CUI* , WEIDONG LI, HURICHA JIN, JIANBO FAN, YUYU SUN and ZHIMING CUI
}

Department of Spine Surgery, The Second Affiliated Hospital of Nantong University, Nantong, Jiangsu 226001, P.R. China

Received August 17, 2019; Accepted January 30, 2020

DOI: $10.3892 /$ etm.2020.8555

\begin{abstract}
Facet joint osteoarthritis (FJOA) is a common degenerative joint disorder with high prevalence in the elderly. FJOA causes lower back pain and lower extremity pain, and thus severely impacts the quality of life of affected patients. Emerging studies have focused on the histomorphological and histomorphometric changes in FJOA. However, the dynamic genetic changes in FJOA have remained to be clearly determined. In the present study, previously obtained RNA deep sequencing data were subjected to an ingenuity pathway analysis (IPA) and canonical signaling pathways of differentially expressed genes (DEGs) in FJOA were studied. The top 25 enriched canonical signaling pathways were identified and canonical signaling pathways with high absolute values of z-scores, specifically leukocyte extravasation signaling, Tec kinase signaling and osteoarthritis pathway, were investigated in detail. DEGs were further categorized by disease, biological function and toxicity (tox) function. The genetic networks between DEGs as well as hub genes in these functional networks were also investigated. It was demonstrated that $\mathrm{C}-\mathrm{X}-\mathrm{C}$ motif chemokine ligand 8, elastase, neutrophil expressed, growth factor independent 1 transcriptional repressor, Spi-1 proto-oncogene, CCAAT enhancer binding protein epsilon, GATA binding protein 1, TAL bHLH transcription factor 1, erythroid differentiation factor, minichromosome maintenance complex component 4, BTG anti-proliferation factor 2, BRCA1 DNA repair-associated, cyclin D1, chromatin assembly factor 1 subunit A, triggering receptor expressed on myeloid cells 1 and tumor protein p63 were hub genes in
\end{abstract}

Correspondence to: Mr. Yuyu Sun or Dr Zhiming Cui, Department of Spine Surgery, The Second Affiliated Hospital of Nantong University, 6 Hai'er Alley North, Chongchuan, Nantong, Jiangsu 226001, P.R. China

E-mail: syy_spine@126.com

E-mail: czmspine@126.com

${ }^{*}$ Contributed equally

Key words: facet joint osteoarthritis, RNA deep sequencing, bioinformatics, ingenuity pathway analysis, network, canonical signaling pathways, diseases and functions, tox functions, hub gene the top 5 IPA networks (with a score $>30$ ). The present study provides insight into the pathological processes of FJOA from a genetic perspective and may thus benefit the clinical treatment of FJOA.

\section{Introduction}

Facet joint osteoarthritis (FJOA) is a common degenerative joint disorder that causes the degeneration and breakdown of cartilage and restricts the movement of joints (1). It has been reported that lumbar FJOA occurs at high prevalence and the presence of FJOA is highly associated with age $(2,3)$. A community-based cross-sectional study indicated that in populations aged $<50$ years, the prevalence of FJOA was $<45 \%$, while it was $\sim 75 \%$ in populations aged $>50$ years (4). FJOA generally causes lower back pain and lower extremity pain and thus largely limits patients' daily activities and severely impacts their quality of life (5). At present, FJOA is generally treated with non-steroidal anti-inflammatory drugs to reduce inflammation and diminish lower back pain (6). However, the therapeutic effects of FJOA are far from satisfactory. The identification of novel clinical treatments for FJOA requires a deeper understanding of FJOA from the cellular and molecular aspects.

The pathological process of FJOA involves the degeneration of a variety of tissues, including cartilage, bones, capsules and synovial tissues (7). Histomorphological and histomorphometric studies indicated that patients with FJOA exhibited thinning or even loss of cartilage and subchondral bones, absence of osteophyte formation, loss of proteoglycans, increased apoptosis of chondrocytes and clustering of chondrocytes (8-10). Histopathological studies of subchondral bone and marrow compartments of osteoarthritic facet joints revealed that patients with FJOA possessed extensive de novo bone and blood vessel formation in subchondral bone tissues and increased inflammatory cell infiltration, as well as enhanced osteoclast activity in subchondral marrow spaces (7). A comparative study of healthy and osteoarthritic lumbar facet joints demonstrated that the thicknesses and the porosities of subchondral cortical plates in patients with FJOA were not as good as those in the healthy population (3). Furthermore, molecular investigations revealed the infiltration of inflammatory cells, increased amounts of pro-inflammatory factors [Including growth-regulated oncogene $\alpha$, soluble intercellular adhesion molecule 1 , interferon $\gamma$, interleukin (IL)- $1 \beta$, 
IL-17, chemokine (C-C motif) ligand 5, tumor necrosis factor $\alpha$, IL- $1 \alpha$ and IL-17E], and increased amounts of anti-inflammatory cytokines, including IL-10 and IL-13 in degenerative facet joint capsular tissues (11).

Although a better understanding of the morphological and molecular changes underlying FJOA has been obtained, the dynamic genetic changes in FJOA have not been well elucidated. In a previous study, by combined use of RNA deep sequencing and Database for Annotation Visualization and Integrated Discovery bioinformatics resource, differentially expressed genes (DEGs) in FJOA were screened and enriched Kyoto Encyclopedia of Genes and Genomes (KEGG) pathways were determined, which revealed that the Wnt and $\mathrm{NF}-\kappa \mathrm{B}$ signaling pathways were significantly involved in FJOA (12). In the present study, the Ingenuity Pathway Analysis (IPA) software, an advanced bioinformatics tool with a massive built-in knowledge database, was used to analyze DEG-associated canonical signaling pathways in FJOA. Furthermore, the correlations between differentially expressed genes, diseases and functions (e.g. toxicity functions) were determined. The interactions between DEGs were also investigated.

\section{Materials and methods}

Human tissue collection and RNA deep sequencing. Human facet joint tissue collection and RNA deep sequencing were performed as previously described (12). In brief, healthy facet joint tissues were gathered from a total of 10 patients with vertebral fracture who received internal fixation of the lumbar spine. Pathological facet joint tissues were gathered from a total of 48 patients with FJOA who received lumbar surgery for neurogenic claudication between January and December 2017 at the Second Affiliated Hospital of Nantong University. A total of 21 male patients and 27 female patients were included. The age range was $46-79$ years and the average age was $64 \pm 1.7$ years. In total, 20 out of these 48 patients with FJOA had a facet joint degeneration grading of 2 , and 28 out of these 48 patients had an FJOA grade of 3 . The healthy or pathological facet joint tissues were respectively divided into 3 portions and subjected to RNA deep sequencing using the Illumina Hiseq X10 platform. RNA deep sequencing outcomes revealed balanced base compositions of raw reads and even distributions of bases along reads and on reference genes, indicating that high-quality data were obtained. Fragments per kilobase of exon per million fragments mapped were obtained to quantify the expression levels of mapped genes. All procedures were ethically approved by the Human Ethics Committee of the Second Affiliated Hospital of Nantong University (Nantong, China) and documents of informed consent were signed by the patients.

Bioinformatics analysis. Genes with a $\log _{2}$ fold change $>2$ or $\leq 2$ and a false discovery rate (adjusted P-value) $<0.05$ were considered as differentially expressed and were analyzed by the IPA software (version 2018; Ingenuity Systems; QIAGEN). RNA deep sequencing outcomes were uploaded to IPA for core analysis and jointly analyzed with the global molecular network in the ingenuity pathway knowledge base (IPKB) $(13,14)$. Canonical signaling pathways enriched by the differentially expressed gene were identified and rated according to P-values. The z-scores and ratios of significantly involved canonical signaling pathways were also determined. Z-scores were presented by orange or blue colors and indicated the activation of the canonical signaling pathways. Ratios were calculated by dividing the numbers of DEGs in the canonical signaling pathways to the numbers of total genes in the pathways and indicated the percentages of differentially expressed genes. The outcomes from the IPA core analysis were overlaid with the IPKB in order to identify significantly involved diseases and functions, toxicity (tox) functions and IPA functional networks in FJOA. Tox functions are used in combination with tox lists to link experimental data to clinical pathology endpoints, understand pharmacological response and support mechanism of action and mechanism of toxicity hypothesis generation in IPA software.

Reverse transcription-quantitative $(R T-q) P C R$. The same facet joint tissues used for RNA deep sequencing were subjected to RT-qPCR analysis. Total RNA was isolated from facet joint tissues by using TRIzol reagent (Thermo Fisher Scientific, Inc.) and reversely transcribed to complementary DNA using the Prime-Script reagent kit (Takara Biotechnology Co., Ltd.). qPCR was performed by using SYBR Green Premix Ex Taq (Takara Biotechnology Co., Ltd.) on an Applied Biosystems StepOne real-time PCR System (Thermo Fisher Scientific, Inc.). The amplification reaction protocol was as follows: A total of 40 cycles at $95^{\circ} \mathrm{C}$ for $5 \mathrm{sec}$ and $60^{\circ} \mathrm{C}$ for $10 \mathrm{sec}$. Relative quantification was performed by using the comparative $2^{-\Delta \Delta C q}$ method (15) with GAPDH as the reference gene. The sequences of all primer pairs are listed in Table I.

Statistical analysis. The PCR results are expressed as the mean \pm standard error of the mean. The differences between the FJOA group and the healthy control group were assessed by using Student's t-test. Statistical analysis was performed with GraphPad Prism 5.0 (GraphPad Software, Inc.). P<0.05 was considered to indicate a statistically significant difference.

\section{Results}

Identification of significantly enriched canonical signaling pathways. In order to investigate significantly involved signaling pathways involved in FJOA, outcomes from RNA deep sequencing were subjected to IPA canonical signaling pathway analysis. DEGs in FJOA were categorized according to these canonical signaling pathways. The activated canonical signaling pathways in FJOA were screened and are listed in Table SI. After rating the identified canonical signaling pathways according to their P-values, the top 25 canonical signaling pathways in FJOA were identified (Fig. 1). The ratios of DEGs to total genes in these signaling pathways were also listed.

The top enriched canonical signaling pathways were mainly assigned to the following categories: Cellular stress and injury [glycoprotein VI platelet (GP6) signaling pathway and osteoarthritis pathway]; disease-specific pathways (role of osteoblasts, osteoclasts and chondrocytes in rheumatoid arthritis; atherosclerosis signaling; hepatic fibrosis/hepatic stellate cell activation; role of macrophages, fibroblasts and endothelial cells in rheumatoid arthritis; and role of IL-17A 
Table I. List of PCR primers.

Gene/primer Sequence (5' to 3')

\begin{tabular}{|c|c|}
\hline \multicolumn{2}{|l|}{ ELANE } \\
\hline Forward & CAGGCATCTGCTTCGGGGAC \\
\hline Reverse & AGGGGCGAAGGCATCTGGGT \\
\hline \multicolumn{2}{|l|}{ BTG2 } \\
\hline Forward & СТCCATCTGCGTCTTGTACGA \\
\hline Reverse & AGACTGCCATCACGTAGTTCT \\
\hline \multicolumn{2}{|l|}{ BRCA1 } \\
\hline Forward & AGCGCCAGTCATTTGCTCCG \\
\hline Reverse & GGACCCAGAGTGGGCAGAGAATG \\
\hline \multicolumn{2}{|l|}{ CHAF1A } \\
\hline Forward & GCAGGCATCTGTCAATTAAG \\
\hline Reverse & AGCATCTGACTAGCAACCAC \\
\hline \multicolumn{2}{|l|}{ ТР63 } \\
\hline Forward & ACAGGAAGGCGGATGAAGAT \\
\hline Reverse & TGTGTGCTGAGGAAGGTACT \\
\hline \multicolumn{2}{|l|}{ CXCL8 } \\
\hline Forward & AATGAAAAGATGAGGGTGCAT \\
\hline Reverse & GCTTGTGTGCTCTGCTGTCT \\
\hline \multicolumn{2}{|l|}{ GFI1 } \\
\hline Forward & TCCCTGTCAGTACTGTGGCA \\
\hline Reverse & TGGAGCTCTGACTGAAGGCT \\
\hline \multicolumn{2}{|l|}{ CCND1 } \\
\hline Forward & СССААСААСТТССТСТССТ \\
\hline Reverse & TCCAGAAGGGCTTCAATCTG \\
\hline \multicolumn{2}{|l|}{ SPI1 } \\
\hline Forward & GACACGGATCTATACCAACGCC \\
\hline Reverse & CCGTGAAGTTGTTCTCGGCGAA \\
\hline \multicolumn{2}{|l|}{ CEBPE } \\
\hline Forward & AGTACCAAGTGGCACACTGC \\
\hline Reverse & GAGAAGGGGACTGCAGGGA \\
\hline \multicolumn{2}{|l|}{ GATA1 } \\
\hline Forward & CAGGGCAGAATCCACAAACT \\
\hline Reverse & TCCTCTGCATCAACAAGCC \\
\hline \multicolumn{2}{|l|}{ TAL1 } \\
\hline Forward & AACAACAACCGGGTGAAGAG \\
\hline Reverse & CATTCACATTCTGCTGCCTC \\
\hline \multicolumn{2}{|l|}{ MCM4 } \\
\hline Forward & GTATTTTTTGGTAGAGACGGCTTC \\
\hline Reverse & GTGACGTGGGTCGGAAAC \\
\hline \multicolumn{2}{|l|}{ TREM1 } \\
\hline Forward & AAGGAGCCTCACATGCTGTT \\
\hline Reverse & CACAGTTCTGGGGCTGGTAT \\
\hline \multicolumn{2}{|l|}{ GAPDH } \\
\hline Forward & CCAAGGTCATCCATGACAAC \\
\hline Reverse & TGTCATACCAGGAAATGAGC \\
\hline
\end{tabular}

in psoriasis); cellular immune response [granulocyte adhesion and diapedesis; leukocyte extravasation signaling; agranulocyte adhesion and diapedesis; Fc $\gamma$ receptor-mediated phagocytosis in macrophages and monocytes; triggering receptor expressed on myeloid cells 1 (TREM1) signaling; phagosome formation; natural killer cell signaling; $\mathrm{T}$ cell receptor signaling; primary immunodeficiency signaling; inducible T-cell costimulator (iCOS)-iCOS ligand (iCOSL) signaling in T helper cells; CD28 signaling in T-helper cells; and IL-8 signaling]; cell cycle regulation (estrogen-mediated S-phase entry and growth arrest and DNA damage-inducible $\alpha$ signaling); intracellular and second messenger signaling (Tec kinase signaling); pathogen-influenced signaling (virus entry via endocytic pathways); biosynthesis (Heme biosynthesis II); and organismal growth and development (human embryonic stem cell pluripotency).

Besides the P-values of these canonical signaling pathways, $\mathrm{z}$-scores and the IPA-calculated indexes of the functional significances of these canonical signaling pathways were also determined. Z-scores were presented in orange or blue colors. Orange color indicated that the results of differentially expressed gene-associated signaling pathways were consistent with the original prediction, whereas blue indicated that results were opposite to the original prediction. The saturation of the color of each signaling pathway reflected the value of the z-score. It was observed that the following signaling pathways had z-scores that indicated their functional importance in FJOA: GP6 signaling pathway, leukocyte extravasation signaling, Fc $\gamma$ receptor-mediated phagocytosis in macrophages and monocytes, TREM1 signaling, estrogen-mediated S-phase entry, iCOS-iCOSL signaling in T helper cells, Tec kinase signaling, osteoarthritis pathway, CD28 signaling in T helper cells and IL-8 signaling.

Leukocyte extravasation signaling. Canonical signaling pathways with high solute values of z-scores were investigated in detail. Leukocyte extravasation, also known as diapedesis, is the movement of leukocytes out of the circulatory system and the migration of leukocytes towards injured or infected tissue. The process of leukocyte extravasation includes a first contact between leukocytes and endothelial cells, the rolling adhesion and tight adhesion of leukocytes along the inner surface of the vessel wall, as well as the transmigration of leukocytes through spaces between endothelial cells. A schematic diagram of the leukocyte extravasation signaling is presented in Fig. 2, with the DEGs in the canonical signaling pathway labeled by different colors. Genes whose expressions were upregulated in FJOA were labeled in red and genes whose expressions were downregulated in FJOA were labeled in green. It was observed that various molecules, particularly numerous cellular adhesion molecules, including integrin $\alpha$ (ITGA) and ITGB subunits, were downregulated in FJOA.

Tec kinase signaling. Another canonical signaling pathway with a high solute value of the z-score, Tec kinase signaling, was also studied. The tyrosine-protein kinase Tec is an enzyme that belongs to the Tec family of non-receptor protein-tyrosine kinases. Tec kinase is activated under various conditions, for instance by the activation of $\mathrm{G}$ protein $\alpha$ subunit, the activation of focal adhesion kinase, the binding of cytokine to cytokine receptor, the activation of the death receptor, the activation of receptor tyrosine kinase, the binding of antigen to the T-cell receptor, the binding of IgE complex to IgE-loaded high-affinity 


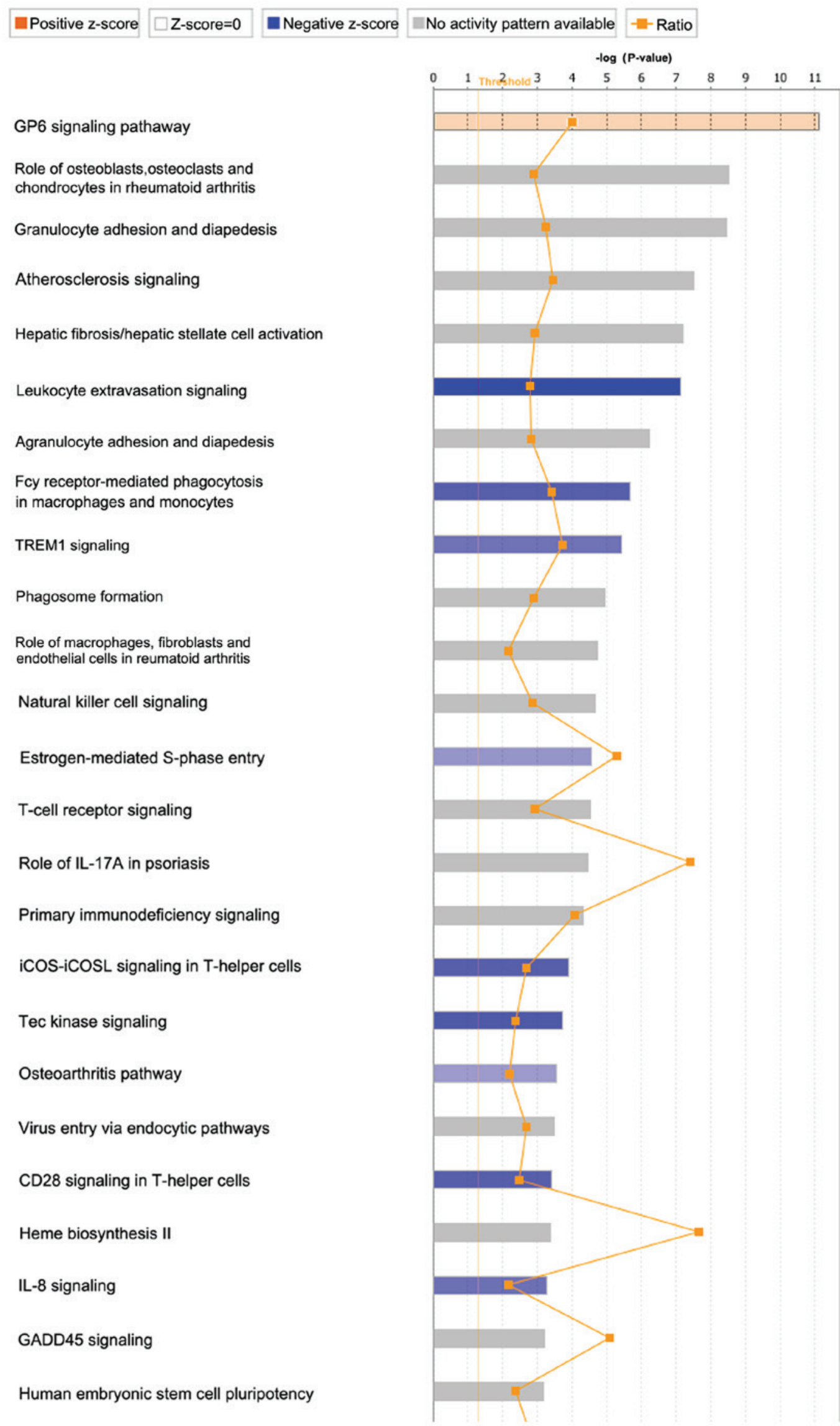

Figure 1. Top 25 canonical signaling pathways in facet joint osteoarthritis. The-log (P-value), z-score and ratio of top 25 significantly activated canonical signaling pathways are listed. TREM1, triggering receptor expressed on myeloid cells 1; GP6, glycoprotein VI platelet; IL, interleukin; ICOSL, inducible T-cell costimulator ligand; GADD45A, growth arrest and DNA damage inducible $\alpha$. A scale from light blue to dark blue indicates the level of activation of the canonical signaling pathways. 


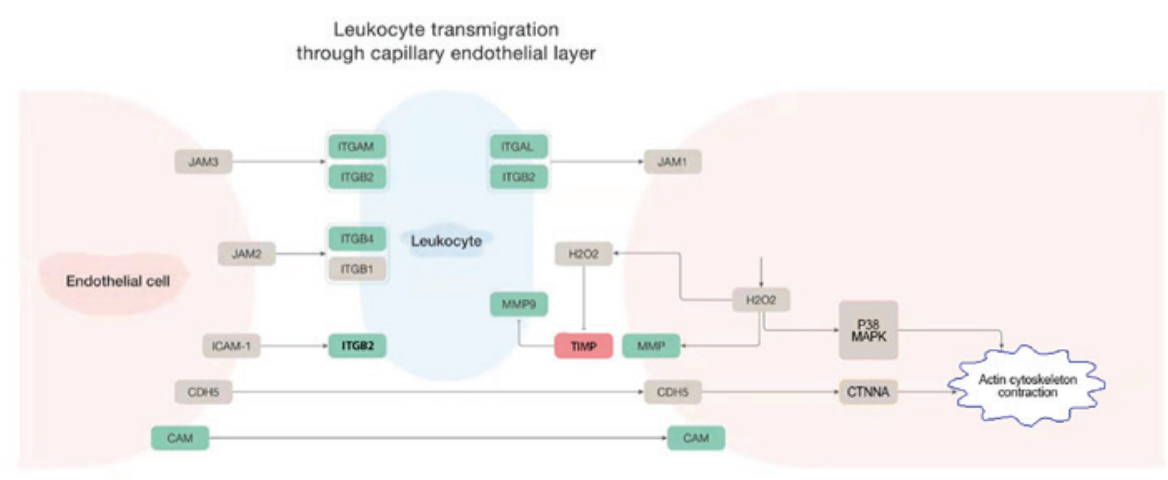

Figure 2. Leukocyte extravasation signaling. A schematic network of leukocyte extravasation signaling modified based on ingenuity pathway analysis software. Upregulated genes were labeled in red and downregulated genes were labeled in green. CAM, KRIT1 ankyrin repeat containing; CDH5, cadherin 5; CTNNA, catenin $\alpha 1$; ICAM, intercellular adhesion molecule; ITGAM, integrin subunit $\alpha M$; ITGB2, integrin subunit $\beta 2$; ITGB4, integrin subunit $\beta 4$; ITGAL, integrin subunit $\alpha \mathrm{L}$; JAM, junctional adhesion molecule; MAPK, mitogen-activated protein kinase; MMP9, matrix metallopeptidase 9; TIMP, TIMP metallopeptidase inhibitor 1.

$\operatorname{IgE}$ receptor and the binding of endotoxin lipopolysaccharide to Toll-like receptor 4 . Activated Tec kinase functions as a second messenger, activates downstream cascades and regulates a large number of physiological processes, including cell adhesion, cell migration, cell apoptosis, actin reorganization, gene expression and transformation (Fig. 3).

Osteoarthritis signaling. Besides the two aforementioned canonical signaling pathways with high solute z-score values, the osteoarthritis signaling was also examined, since FJOA is a type of osteoarthritis that specifically occurs in facet joint tissue. Osteoarthritis is a progressive degradative disease of joints that may be caused by numerous factors, including inflammation, obesity, injury, aging and improper or lack of exercise. These risk factors may severely affect chondrocytes by mediating numerous cytokines and inflammatory signals, including transforming growth factor $\beta$ (TGF- $\beta$ ), IL-1 $\beta$ and IL-8. Certain molecules, e.g. IL-8, activate transcription factor SOX-9, a protective and stabilizing factor, whereas TGF- $\beta$ affects Runt-related transcription factor 2 (a pro-development, pro-degradation and pro-osteoarthritis factor; Fig. 4).

Identification of significantly involved diseases and functions and tox functions. DEGs were shown to be further associated with diseases and functions by using the IPA core analysis. A total of 60 associated diseases and functions were identified in FJOA. These associated diseases and functions were then rated according to their significance (P-value) and the top 20 enriched categories of diseases and functions in FJOA were selected (Fig. 5).

It was demonstrated that cell and tissue growth-associated biological functions, including cellular growth and proliferation, lymphoid tissue structure and development, cellular development, cellular development and hematological system development and function were significant in FJOA. Cellular movement and cell death and survival, two biological functions of cellular behavior, were also enriched. Besides these critical biological processes, it was indicated that the DEGs in FJOA were significantly involved in numerous diseases, including connective tissue disorders, organismal injury and abnormalities, skeletal and muscular disorders, cancer, derma- tological diseases and conditions, respiratory disease and reproductive system disease. Furthermore, it was demonstrated that diseases and functions associated with inflammation and immune responses, including inflammatory disease, inflammatory response, immunological disease, infectious disease and immune cell trafficking, were also significantly involved in FJOA.

Next, the associations between DEGs and IPA toxicity (tox) functions were determined. Following examination of toxicity phenotypes, a total of 47 tox functions were identified. Among the 47 tox functions, 22 exhibited a $\mathrm{P}<0.05$. The top enriched tox function, liver hyperplasia/hyperproliferation, had the lowest P-value of $<7 \times 10^{-11}$. The top 20 tox functions in FJOA are listed (Fig. 6). The tox function analysis, from the genetic aspect, indicated the possible associations of FJOA with other diseases.

Identification of essential functional networks. DEGs in different diseases and functions were further connected with each other to build IPA functional networks. A total of 25 IPA networks were identified. These IPA networks were ranked based on their scores (Table II). The top-scoring IPA network included a total of 34 DEGs and had a score of 33, indicating that the possibility that genes in the IPA network were not connected was not lower the 10 and not higher than 3310 . Even the IPA network with the lowest score had a score of 15, suggesting that these genes were highly connected.

A total of 5 IPA networks exhibited a score of $>30$ (network 1 and 2: Score of 33; network 3, 4 and 5: Score of 31). Numerous significantly enriched diseases and functions were also enriched in these IPA networks. It was indicated that cell and tissue growth-associated diseases and functions, including hematological system development and function, developmental disorder, cellular movement, DNA replication, recombination, repair, cell cycle, and cellular assembly and organization were involved in these top 5 IPA networks. These outcomes suggested that the dynamic changes of cellular development were essential characteristics in FJOA.

These highly significant IPA networks were further outlined in detail (Fig. 7). It was observed that in IPA network 1 , most genes were connected with $\mathrm{C}-\mathrm{X}-\mathrm{C}$ motif 


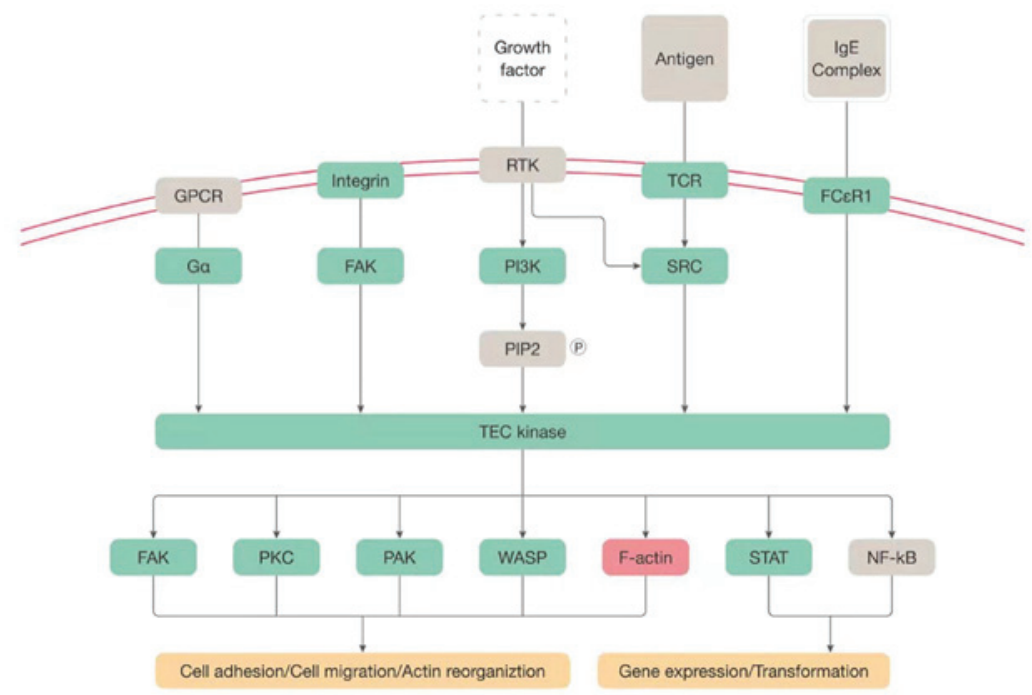

Figure 3. Tec kinase signaling. A schematic network of Tec kinase signaling modified based on ingenuity pathway analysis software. Upregulated genes were labeled in red and downregulated genes were labeled in green. F-actin, filamentous actin; FAK, protein tyrosine kinase 2; FCER1, high affinity immunoglobulin E receptor 1; GPCR, G-protein coupled receptor; IgE, immunoglobulin E; PAK, serine/threonine protein kinase PAK; PI3K, phosphatidylinositol-4,5-bisphosphate 3-kinase; PKC, protein kinase C family; SRC, SRC non-receptor tyrosine kinase family; Tec, tyrosine protein kinase Tec; WASP, WASP actin nucleation promoting factor; TCR, T-cell receptor; Ga, $\alpha$ subunit of heterotrimeric G protein; TEC kinase, Tyrosine-protein kinase; STAT, signal transducer and activator of transcription protein.

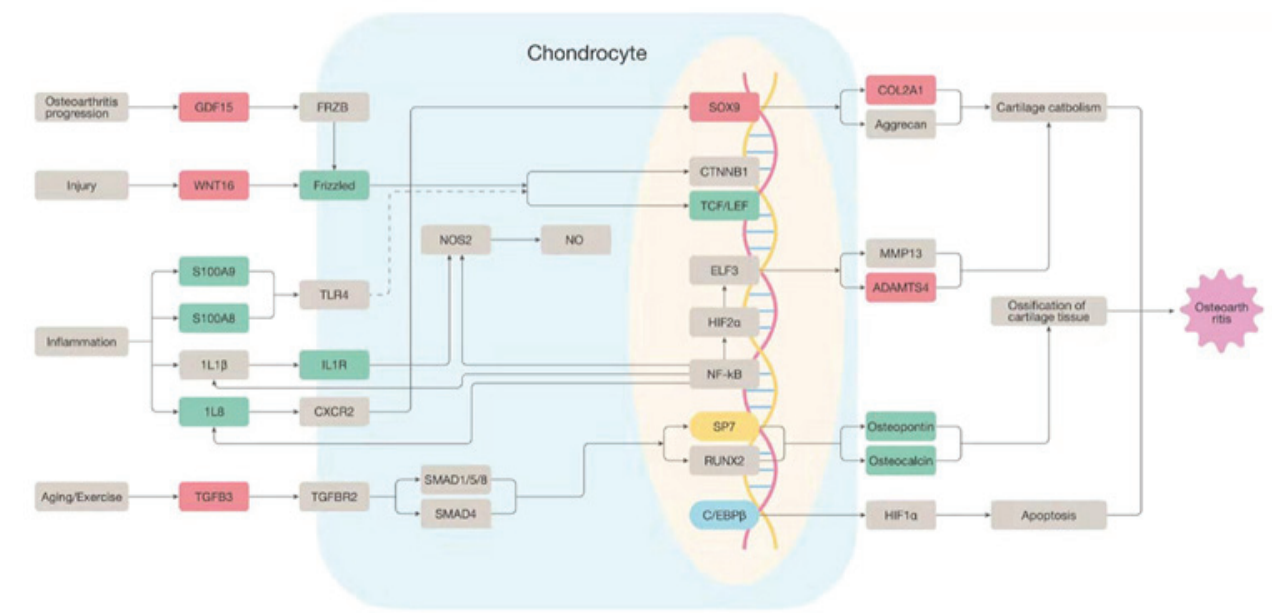

Figure 4. Osteoarthritis signaling. A schematic network of osteoarthritis signaling modified based on ingenuity pathway analysis software. Upregulated genes were labeled in red and downregulated genes were labeled in green. GDF15, Growth Differentiation Factor 15; WNT16, Wnt Family Member 16; Frizzled, frizzled gene family; S100A9, S100 Calcium Binding Protein A9; S100A8, S100 Calcium Binding Protein A8; IL8, Interleukin 8; IL1R, Interleukin 1 Receptor Type 1; TGFB3, Transforming Growth Factor Beta 3; SOX9, SRY-Box Transcription Factor 9; TCF/LEF, Transcription Factor/Lymphoid Enhancer Binding Factor; SP7, Sp7 Transcription Factor; COL2A1, Collagen Type II Alpha 1 Chain; ADAMTS4, ADAM Metallopeptidase With Thrombospondin Type 1 Motif 4.

chemokine ligand 8 (CXCL8). Furthermore, elastase, neutrophil expressed (ELANE), growth factor independent 1 transcriptional repressor (GFI1), Spi-1 proto-oncogene (SPI1), CCAAT/enhancer binding protein epsilon (CEBPE) and GATA binding protein 1 (GATA1) were also connected with many other DEGs (Fig. 7A). In IPA network 2, hub genes were TAL BHLH transcription factor 1, erythroid differentiation factor (TAL1), minichromosome maintenance complex component 4 (MCM4), BTG anti-proliferation factor 2 (BTG2) and BRCA1, DNA repair associated (BRCA1) (Fig. 7B). In IPA network 3, the most connected gene was cyclin D1 (CCND1) (Fig. 7C). Chromatin assembly factor 1 subunit $\mathrm{A}$ (CHAF1A) and histones were hub genes in IPA network 4 (Fig. 7D), whereas TREM1 and tumor protein P63 (TP63) were hub genes in IPA network 5 (Fig. 7E).

The expression levels of certain central genes identified, including ELANE, BTG2, BRCA1, CHAF1A, TP63, CXCL8, GFI1, CCND1, SPI1, CEBPE, GATA1, TAL1, MCM4 and TREM1, were further examined by RT-qPCR. The results indicated that, consistent with the sequencing results, most of these genes were dysregulated in lesioned facet joint tissue samples collected from patients with FJOA compared with those in the healthy facet joint tissue samples (Fig. 8). 


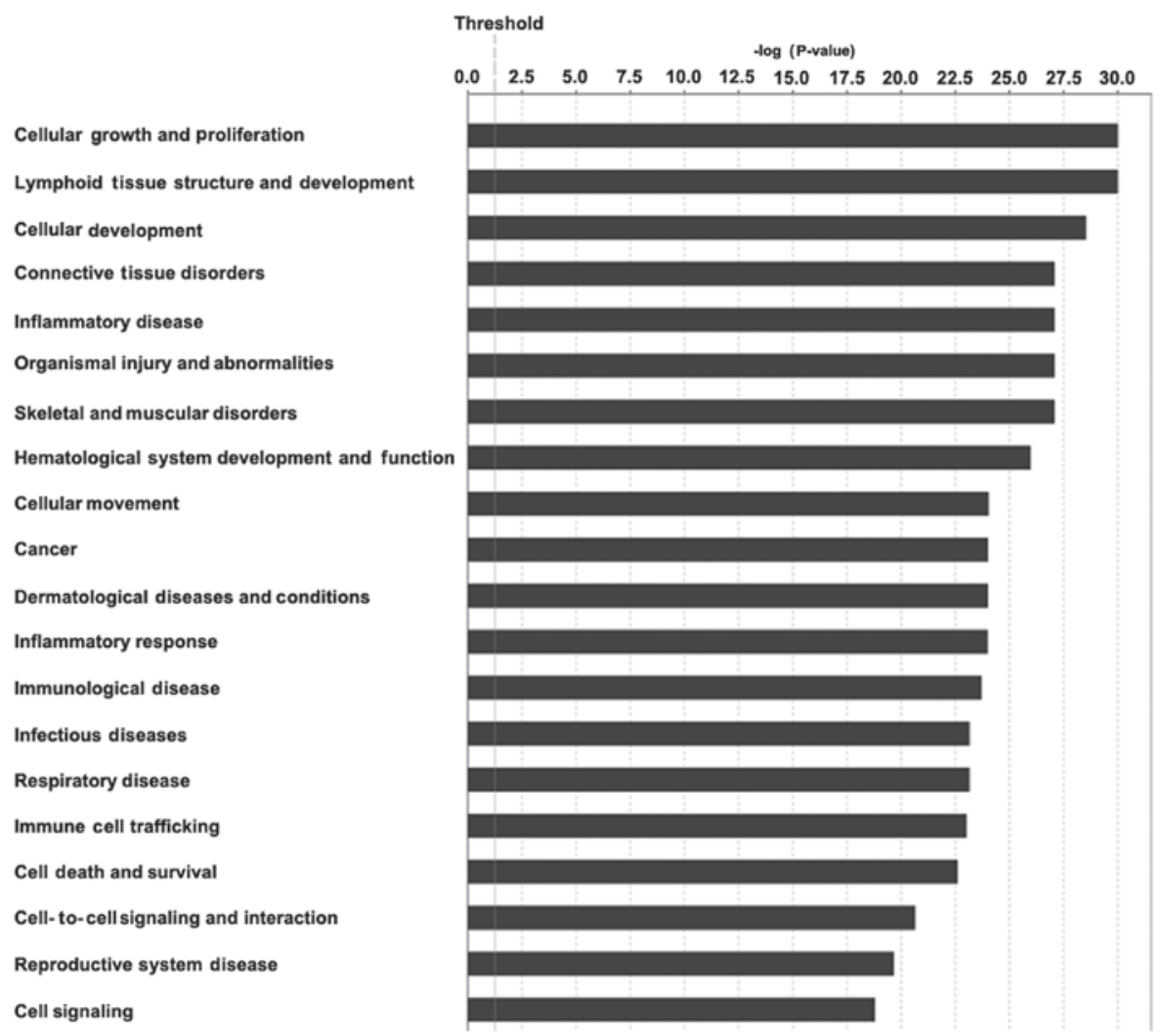

Figure 5. Top 20 diseases and functions in facet joint osteoarthritis. The-log (P-value) of the top 20 significantly involved diseases and functions is listed.

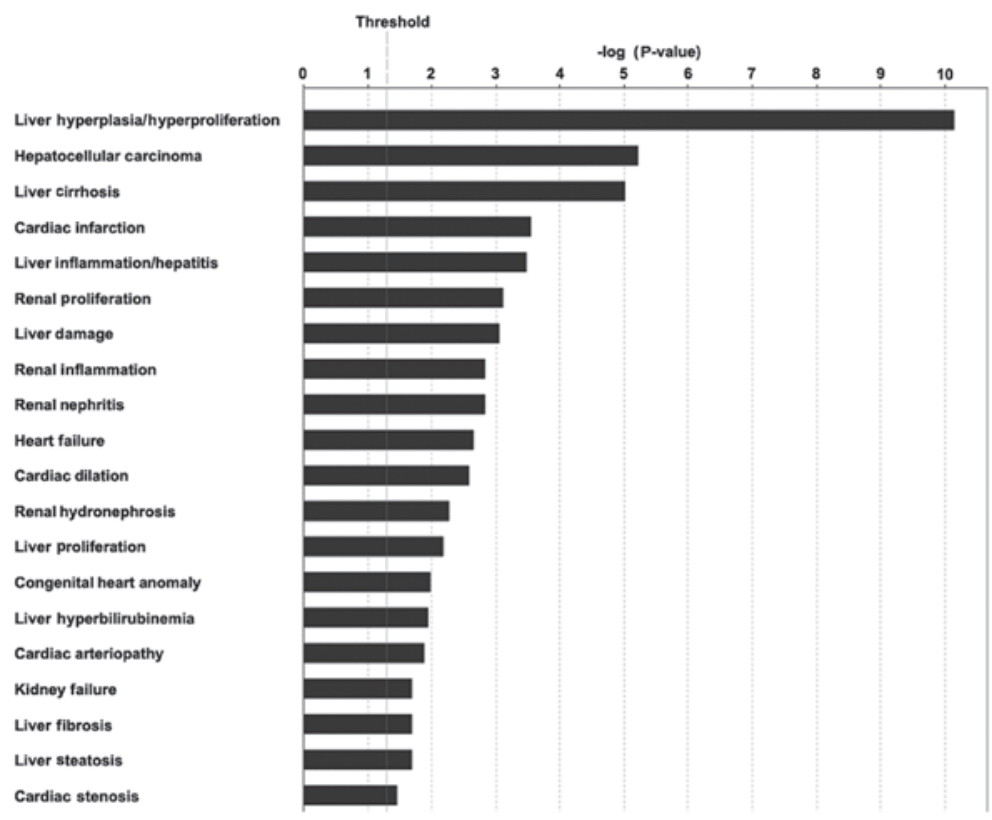

Figure 6. Top 20 tox functions in facet joint osteoarthritis. The-log (P-value) of the top 20 significantly involved tox functions is listed.

\section{Discussion}

In the present study, a high-resolution analysis of transcriptional profiling was performed to characterize the changes in expression in FJOA and IPA core analysis was used to interpret gene expression profiles. IPA has been broadly used in the interpretation of high-throughput data, including
RNA sequencing, small RNA sequencing, DNA sequencing, microarray, metabolomics, proteomics and small-scale experiments (16). By using the built-in literature-supported IPKB database, the global molecular network in numerous physiological and pathological processes may be searched and discovered. In the present study, by using IPA core analysis, DEGs in FJOA were categorized into IPA canonical signaling 
Table II. Ingenuity pathway analysis networks in facet joint osteoarthritis.

\begin{tabular}{|c|c|}
\hline Score & Top diseases and functions \\
\hline 33 & $\begin{array}{l}\text { Cell-to-cell signaling and interaction, hematological system development and function, immune cell } \\
\text { trafficking }\end{array}$ \\
\hline 33 & Cardiovascular disease, developmental disorder, hematological disease \\
\hline 31 & Cell cycle, cellular movement, cancer \\
\hline 31 & DNA replication, recombination, and repair, cell cycle, cellular assembly and organization \\
\hline 31 & Connective tissue disorders, dermatological diseases and conditions, developmental disorder \\
\hline 27 & Antimicrobial response, inflammatory response, cell-to-cell signaling and interaction \\
\hline 27 & Cardiovascular disease, cell death and survival, connective tissue disorders \\
\hline 27 & Cancer, connective tissue disorders, organismal injury and abnormalities \\
\hline 27 & $\begin{array}{l}\text { Cell-to-cell signaling and interaction, hematological system development and function, cellular } \\
\text { development }\end{array}$ \\
\hline 25 & $\begin{array}{l}\text { Hematological system development and function, cell-to-cell signaling and interaction, cell } \\
\text { morphology }\end{array}$ \\
\hline 25 & Cell cycle, reproductive system development and function, cell death and survival \\
\hline 25 & Cell signaling, molecular transport, vitamin and mineral metabolism \\
\hline 23 & $\begin{array}{l}\text { Cell-to-cell signaling and interaction, hematological system development and function, immune cell } \\
\text { trafficking }\end{array}$ \\
\hline 23 & Cell cycle, organismal injury and abnormalities, reproductive system disease \\
\hline 23 & $\begin{array}{l}\text { Hematological system development and function, humoral immune response, lymphoid tissue struc- } \\
\text { ture and development }\end{array}$ \\
\hline 22 & Cellular movement, immune cell trafficking, hematological system development and function \\
\hline 20 & $\begin{array}{l}\text { Cellular movement, cell-to-cell signaling and interaction, hematological system development and } \\
\text { function }\end{array}$ \\
\hline 20 & Cell cycle, cellular assembly and organization, DNA replication, recombination and repair \\
\hline 20 & Cancer, organismal injury and abnormalities, reproductive system disease \\
\hline 19 & Cellular development, cellular movement, cell morphology \\
\hline 19 & DNA replication, recombination, and repair, nucleic acid metabolism, small molecule biochemistry \\
\hline 17 & $\begin{array}{l}\text { Cell-to-cell signaling and interaction, hematological system development and function, immune cell } \\
\text { trafficking }\end{array}$ \\
\hline 17 & Cellular movement, hematological system development and function, immune cell trafficking \\
\hline 15 & Cellular assembly and organization, DNA replication, recombination and repair, cancer \\
\hline 15 & Infectious diseases, cell signaling, molecular transport \\
\hline
\end{tabular}

pathways and the top 25 enriched IPA canonical signaling pathways in FJOA were identified.

These top enriched canonical signaling pathways were mainly categorized into cellular stress and injury-associated pathways, disease-specific pathways, cellular immune response-associated pathways, cell cycle regulation-associated pathways, intracellular and second messenger signaling, pathogen-influenced signaling, biosynthesis-associated pathways, and organismal growth and development-associated pathways. From the aspect of the number of signaling pathways involved, cellular immune response appeared to be most significant in FJOA, since nearly half of the canonical signaling pathways (12 out of 25) were cellular immune response-associated pathways. Inflammation and immune responses are generally considered as key components of osteoarthritis (17-19). It was identified that numerous cell types in joints, including synovial cells and articular chondrocytes, may express inflammatory mediators (20). The modulation of immune response was even regarded as a novel and effective treatment of osteoarthritis (21). On the other hand, the specific roles of inflammation and immune responses in FJOA, a universal form of lumbar osteoarthritis, were not well demonstrated. The IPA analysis, from the aspect of genetic expression, demonstrated the importance of inflammation and immune responses in FJOA. The observations of the present study were also consistent with outcomes from a previous KEGG pathway analysis, which indicated that Wnt signaling and NF- $\kappa \mathrm{B}$ signaling pathways were deeply involved in the process of facet joint degeneration (12). Furthermore, it is worth noting that it has been well elucidated that aging is closely associated with dysregulated inflammation and immune responses (22-24). Since FJOA is also highly associated with aging, it is expected that inflammation and immune responses may be critical causes of FJOA.

Besides the integral analysis of IPA canonical signaling pathways, the canonical signaling pathways with high absolute values of z-scores were selected, DEGs in FJOA in these signaling pathways were identified and the expression levels of 
A

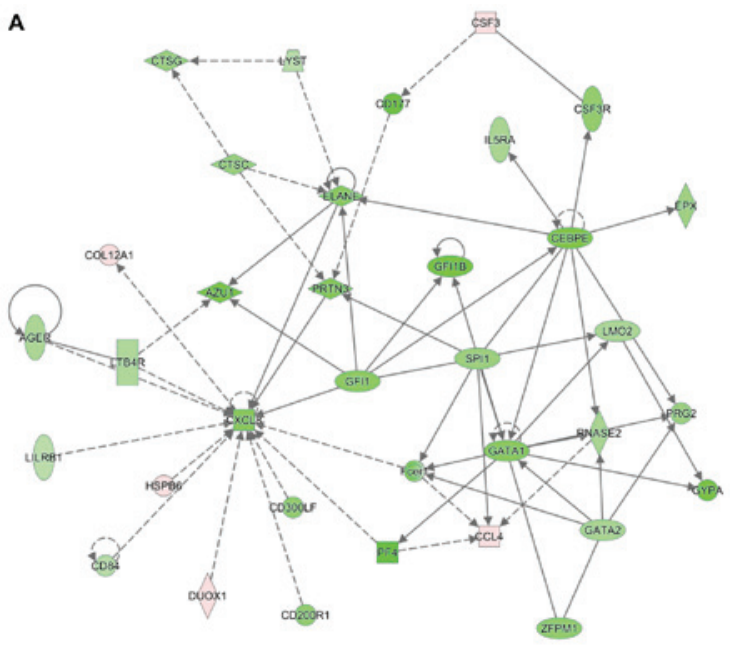

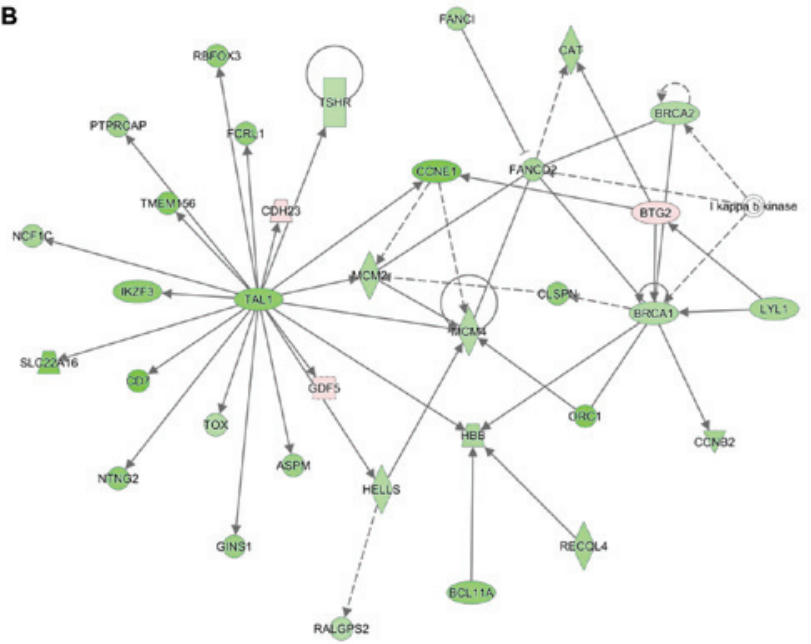
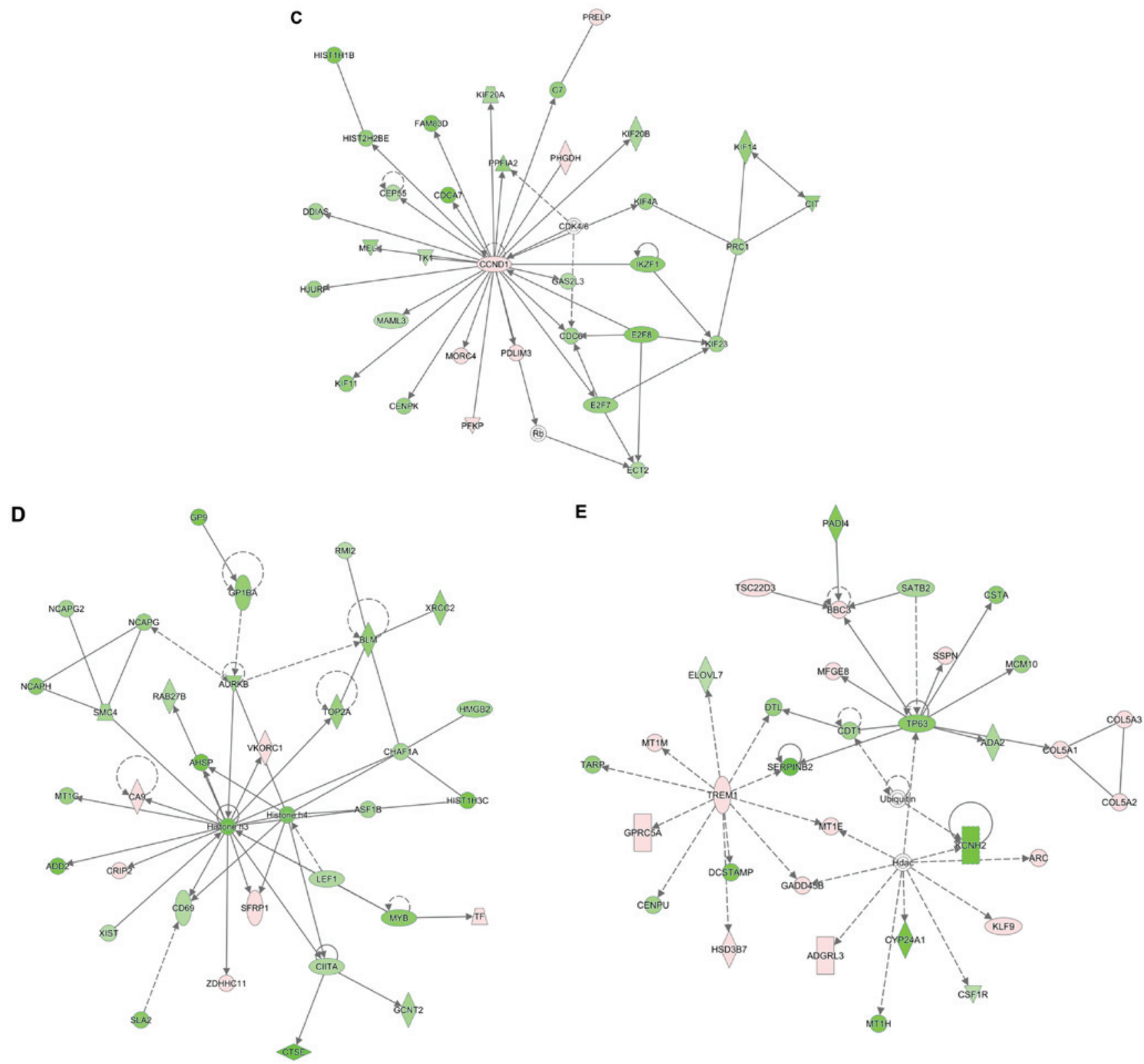

Figure 7. Top 5 ingenuity pathway analysis networks in facet joint osteoarthritis. Upregulated genes are labeled in red, while downregulated genes are labeled in green. The saturation of color is correlated with the fold change of the gene (high saturation means high fold change and low saturation means low fold change). Solid lines indicate direct connections, while dotted lines indicate indirect connections (circular arrows means influence itself). Ellipses represent transcription regulators, rhombuses represent enzymes, trapezoids represent transporters, double circles represent a complex/group and circles represent others. (A) CXCL8, ELANE, GFI1, SPI1, CEBPE, GATA1, (B) TAL1, BTG2, BRCA1, (C) CCND1, (D) CHAF1A, histone, (E) TREM1 and TP63 were located in the central positions of IPA networks. 


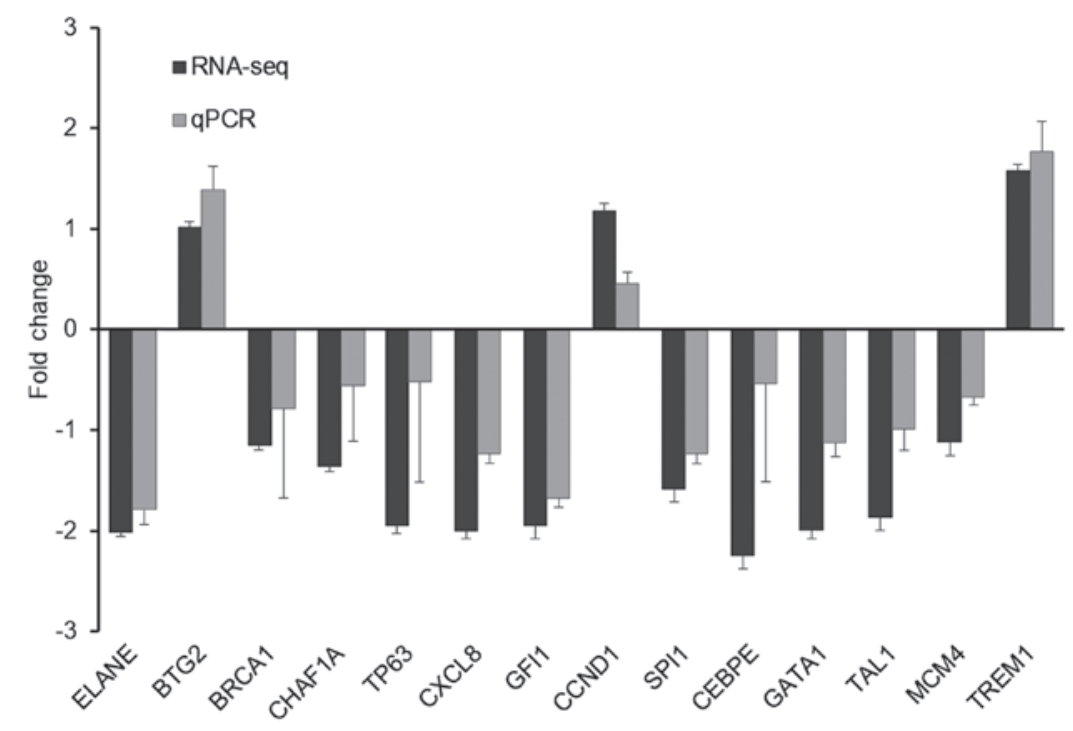

Figure 8. Validation of hub genes in ingenuity pathway analysis networks. The relative mRNA abundance of ELANE, BTG2, BRCA1, CHAF1A, TP63, CXCL8, GFI1, CCND1, SPI1, CEBPE, GATA1, TAL1, MCM4 and TREM1 was determined by reverse transcription-qPCR with normalization to GAPDH. Results are presented as the mean \pm standard deviation from 3 independent experiments. RNA-seq, RNA sequencing; qPCR, quantitative PCR; ELANE, elastase, neutrophil expressed; BTG2, BTG anti-proliferation factor 2; BRCA1, BRCA1 DNA repair-associated; CHAF1A, chromatin assembly factor 1 subunit A; TP63, tumor protein p63; CXCL8, C-X-C motif chemokine ligand 8; GFI1, growth factor independent 1 transcriptional repressor; CCND1, cyclin D1; SPI1, Spi-1 proto-oncogene; CEBPE, CCAAT enhancer binding protein epsilon; GATA1, GATA binding protein 1; TAL1, TAL bHLH transcription factor 1, erythroid differentiation factor; MCM4, minichromosome maintenance complex component 4; TREM1, triggering receptor expressed on myeloid cells 1.

representative genes were validated by RT-qPCR. Leukocyte extravasation signaling is the migration of leukocytes from blood to tissue, a critical step during inflammation. Tec kinase signaling is essential for the development and activation of $\mathrm{B}$ cells and $\mathrm{T}$ cells. Therefore, although Tec kinase signaling is grouped to intracellular and second messenger signaling due to its intercellular localization, Tec kinase signaling is also highly associated with inflammation and immune responses. Similarly, osteoarthritis pathways are also associated with inflammation and immune responses. The diagram of osteoarthritis pathways suggested that osteoarthritis may be elicited and activated by inflammation. Numerous pro-inflammatory and anti-inflammatory factors are also critical in the signaling pathway. Therefore, a detailed study of canonical signaling pathways with high absolute values of z-scores will be explored in future to further clarify the importance of inflammation and immune responses in FJOA. Further studies should also clarify the specific roles of inflammatory mediators in FJOA and the complex interactions between them. The results of the present IPA core analysis of DEGs in FJOA may potentially aid the discovery of inflammatory mediators as novel therapeutic strategies to combat FJOA.

In the present study, the focus was on the biological functions of DEGs in FJOA and the top enriched diseases and functions in FJOA were identified and classified into different categories. Detailed investigation of diseases and functions indicated that cellular behavior-associated biological functions, tissue development-associated diseases and functions, and inflammation and immune response-associated diseases and functions were most significantly enriched in FJOA. These results connected the alterations of genes in FJOA with the pathological features of the condition and indicated that cell development, tissue remodeling, as well as inflammation and immune response, were critical in FJOA. This was also consistent with a previous observation that inflammation-associated signaling pathways were critical in the pathological process of FJOA (12). Furthermore, by categorizing the DEGs into diseases and tox functions, the connections between FJOA and other diseases were also preliminarily revealed and diseases that may be associated with FJOA were identified.

Besides the entire and systematical analysis of significantly involved diseases and functions in FJOA, DEGs in different disease and function categories were connected with one another to build gene-gene interaction and functional networks between differentially expressed genes. Consistent with analytic outcomes from disease and functional categories, cell and tissue growth diseases and functions also involved top diseases and functions in IPA networks. Of the 25 IPA networks in FJOA, the top 5 IPA networks (with a score $>30$ ) were selected for further study.

It was observed that CXCL8, ELANE, GFI1, SPI1, GATA1 (in IPA network 1), TAL1, BTG2 (in IPA network 2), TREM1 (in IPA network 5) were located in the central positions of IPA networks and connected with numerous other differentially expressed genes. The expression levels of these hub genes were validated by RT-qPCR and consistent with RNA-seq and it was confirmed that their expression levels in lesioned facet joint tissue samples collected from patients with FJOA were significantly different from their expression levels in healthy facet joint tissue samples. CXCL8, the hub gene in IPA network 1 , was also a significantly differentially expressed gene in the NF- $\mathrm{KB}$ signaling pathway, an immune and inflammation-associated signaling pathway, as previously demonstrated and validated (12). Likewise, BTG2, the hub gene in IPA network 2, was identified and examined, as it is a critical a mechanosensitive and inflammation-related gene in osteoarthritis (25). The present study suggests that these hub genes may not only exert their biological functions in activated signaling pathways but also affect other genes and signaling pathways. 
Besides CXCL8 and BTG2, certain other hub genes identified in the present study have also been previously studied in osteoarthritis. For instance, quantitative analysis of ELANE, a gene coding for neutrophil elastase, a proteinase secreted by neutrophils and macrophages during inflammation, indicated that the abundance of ELANE mRNA in peripheral blood CD14+ cells from patients with osteoarthritis was lower than that in healthy subjects or in patients with rheumatoid arthritis (26). This was consistent with the present study with regard to the RNA deep sequencing and RT-qPCR results. SPI1, a gene coding for an ETS-domain transcription factor that activates gene expression during myeloid and B-lymphoid cell development, and GATA1, a gene coding for a member of the GATA family of transcription factors, were identified as candidate transcription factors for osteoarthritis $(27,28)$. BRCA1, a gene encoding a tumor suppressor nuclear phosphoprotein that has a role in maintaining genomic stability, was indicated to have a relatively lower abundance in patients with FJOA in the present study; this was in line with the result of a previous study on patients with knee/hip osteoarthritis (29). BRCA1 was also identified as a crucial transcription factor that regulates numerous DEGs located downstream with a role in the development of osteoarthritis (30). Similar to the observations of the present study on FJOA, TREM1, a gene encoding an Ig superfamily receptor that amplifies neutrophil and monocyte-mediated inflammatory responses, was also indicated to be upregulated in patients with osteoarthritis by using microarray gene expression profiling, western blot and immunohistochemical analyses $(31,32)$. A recent study further demonstrated that knockdown of upregulated TREM1 in a mouse model of osteoarthritis inhibited the production of matrix metallopeptidase-13, promote the synthesis of collagen type II, suppress the metabolic imbalance of extracellular matrix and decrease the activity of the NF- $\mathrm{kB}$ signaling pathway (33). The participation of other hub genes identified, including GFI1, CEBPE, TAL1, CHAF1A and TP63, in osteoarthritis, let alone in FJOA, has not been previously determined, to the best of our knowledge. The present study revealed the involvement of these hub genes, indicated their central roles in FJOA and thus proposed potential therapeutic targets for FJOA. The presence of these hub genes in patients with different degrees of FJOA and the biological roles of these hub genes should be further investigated in future studies.

In conclusion, the present study performed an IPA core analysis and a systemic investigation of the transcriptomic signature of FJOA. Diseases, biological functions and tox functions significantly enriched by the DEGs in FJOA, as well as the gene-gene interactions of those DEGs were identified. The present study provides implications for understanding the mechanisms of FJOA and the identification of novel therapeutic approaches for this condition.

\section{Acknowledgements}

Not applicable.

\section{Funding}

This work was supported by The National Natural Science Foundation of China (grant no. 81771319) and Nantong Science and Technology Project (grant no. HS2018002).

\section{Availability of data and materials}

The datasets used and/or analyzed during the current study are available from the corresponding author on reasonable request.

\section{Authors' contributions}

CC, YS and ZC conceived and designed the study. CC, SC, $\mathrm{WL}, \mathrm{HJ}, \mathrm{JF}$ and YS performed the experiments. CC analyzed the data. YS and ZC provided reagents/materials/analysis tools. CC and ZC wrote the manuscript. All authors read and approved the final manuscript.

\section{Ethics approval and consent to participate}

All procedures were ethically approved by The Human Ethics Committee of the Second Affiliated Hospital of Nantong University (Nantong, China) and documents of informed consent were signed by patients.

\section{Patient consent for publication}

Not applicable.

\section{Competing interests}

The authors declare that they have no competing interests.

\section{References}

1. Lewinnek GE and Warfield CA: Facet joint degeneration as a cause of low back pain. Clin Orthop Relat Res: 216-222, 1986.

2. Ko S, Vaccaro AR, Lee S, Lee J and Chang H: The prevalence of lumbar spine facet joint osteoarthritis and its association with low back pain in selected Korean populations. Clin Orthop Surg 6: 385-391, 2014.

3. Netzer C, Distel P, Wolfram U, Deyhle H, Jost GF, Schären S and Geurts J: comparative analysis of bone structural parameters reveals subchondral cortical plate resorption and increased trabecular bone remodeling in human facet joint osteoarthritis. Int J Mol Sci 19: pii: E845, 2018.

4. Kalichman L, Li L, Kim DH, Guermazi A, Berkin V, O'Donnell CJ, Hoffmann U, Cole R and Hunter DJ: Facet joint osteoarthritis and low back pain in the community-based population. Spine (Phila Pa 1976) 33: 2560-2565, 2008.

5. Kim JS, Ahmadinia K, Li X, Hamilton JL, Andrews S, Haralampus CA, Xiao G, Sohn HM, You JW, Seo YS, et al: Development of an experimental animal model for lower back pain by percutaneous injury-induced lumbar facet joint osteoarthritis. J Cell Physiol 230: 2837-2847, 2015.

6. Kim DS, Lee SJ, Park SY, Yoo HJ, Kim SH, Kim KJ and Cho HJ: Differentially expressed genes in rat dorsal root ganglia following peripheral nerve injury. Neuroreport 12: 3401-3405, 2001.

7. Netzer C, Urech K, Hügle T, Benz RM, Geurts J and Schären S: Characterization of subchondral bone histopathology of facet joint osteoarthritis in lumbar spinal stenosis. J Orthop Res 34: 1475-1480, 2016.

8. Bleil J, Maier R, Hempfing A, Schlichting U, Appel H, Sieper J and Syrbe U: Histomorphologic and histomorphometric characteristics of zygapophyseal joint remodeling in ankylosing spondylitis. Arthritis Rheumatol 66: 1745-1754, 2014.

9. Appel H, Maier R, Loddenkemper C, Kayser R, Meier O, Hempfing A and Sieper J: Immunohistochemical analysis of osteoblasts in zygapophyseal joints of patients with ankylosing spondylitis reveal repair mechanisms similar to osteoarthritis. J Rheumatol 37: 823-828, 2010.

10. Eisenstein SM and Parry CR: The lumbar facet arthrosis syndrome. Clinical presentation and articular surface changes. J Bone Joint Surg Br 69: 3-7, 1987. 
11. Kim JS, Ali MH,Wydra F,Li X,Hamilton JL, An HS, Cs-Szabo G, Andrews S, Moric M, Xiao G, et al: Characterization of degenerative human facet joints and facet joint capsular tissues. Osteoarthritis Cartilage 23: 2242-2251, 2015.

12. Chen C, Bao GF, Xu G, Sun Y and Cui ZM: Altered Wnt and $\mathrm{NF}-\kappa \mathrm{B}$ signaling in facet joint osteoarthritis: Insights from RNA deep sequencing. Tohoku J Exp Med 245: 69-77, 2018.

13. $\mathrm{Yu} \mathrm{J}, \mathrm{Gu} \mathrm{X}$ and $\mathrm{Yi}$ S: Ingenuity pathway analysis of gene expression profiles in distal nerve stump following nerve injury: Insights into wallerian degeneration. Front Cell Neurosci 10: 274, 2016.

14. Yi S, Zhang H, Gong L, Wu J, Zha G, Zhou S, Gu X and Yu B: Deep sequencing and bioinformatic analysis of lesioned sciatic nerves after crush injury. PLoS One 10: e0143491, 2015.

15. Livak KJ and Schmittgen TD: Analysis of relative gene expression data using real-time quantitative PCR and the 2(-Delta Delta C(T)) method. Methods 25: 402-408, 2001.

16. Ghosh S, Dutta S, Thorne G, Boston A, Barfield A, Banerjee N, Walker R and Banerjee HN: Core canonical pathways involved in developing human glioblastoma multiforme (GBM). Int J Sci Res Sci Eng Technol 3: 458-465, 2017.

17. Raman S, FitzGerald U and Murphy JM: Interplay of inflammatory mediators with epigenetics and cartilage modifications in osteoarthritis. Front Bioeng Biotechnol 6: 22, 2018.

18. Goldring MB and Goldring SR: Osteoarthritis. J Cell Physiol 213: 626-634, 2007.

19. Ray A and Ray BK: An inflammation-responsive transcription factor in the pathophysiology of osteoarthritis. Biorheology 45 399-409, 2008

20. Shen J, Abu-Amer Y,O'Keefe RJ and McAlinden A: Inflammation and epigenetic regulation in osteoarthritis. Connect Tissue Res 58: 49-63, 2017

21. Fahy N, Farrell E, Ritter T, Ryan AE and Murphy JM: Immune modulation to improve tissue engineering outcomes for cartilage repair in the osteoarthritic joint. Tissue Eng Part B Rev 21: 55-66, 2015.

22. Wu D and Meydani SN: Age-associated changes in immune and inflammatory responses: Impact of vitamin $\mathrm{E}$ intervention. J Leukoc Biol 84: 900-914, 2008.

23. Meydani SN and Wu D: Nutrition and age-associated inflammation: Implications for disease prevention. JPEN J Parenter Enteral Nutr 32: 626-629, 2008.
24. Han SN and Meydani SN: Antioxidants, cytokines, and influenza infection in aged mice and elderly humans. J Infect Dis 182 (Suppl 1): S74-S80, 2000

25. Guo Y, Song Y, Zhang Y and Yang L: Identification of Btg2 As A mechanosensitive gene by functional screening integrative analyses. Mol Cell Biomech 16 (Suppl 2): S119, 2019.

26. Trzybulska D, Olewicz-Gawlik A, Graniczna K, Kisiel K, Moskal M, Cieślak D, Sikora J and Hrycaj P: Quantitative analysis of elastase and cathepsin G mRNA levels in peripheral blood CD14(+) cells from patients with rheumatoid arthritis. Cell Immunol 292: 40-44, 2014

27. Dong S, Xia T, Wang L, Zhao Q and Tian J: Investigation of candidate genes for osteoarthritis based on gene expression profiles. Acta Orthop Traumatol Turc 50: 686-690, 2016.

28. Wang K, Zhao L, Liu X, Hao Z, Zhou Y, Yang C and Li H: Differential co-expression analysis of rheumatoid arthritis with microarray data. Mol Med Rep 10: 2421-2426, 2014.

29. Pellicelli M, Picard C, Wang D, Lavigne P and Moreau A: E2F1 and TFDP1 Regulate PITX1 expression in normal and osteoarthritic articular chondrocytes. PLoS One 11: e0165951, 2016.

30. Fei Q, Lin J, Meng H, Wang B, Yang Y, Wang Q, Su N, Li J and $\mathrm{Li} \mathrm{D}$ : Identification of upstream regulators for synovial expression signature genes in osteoarthritis. Joint Bone Spine 83: 545-551, 2016.

31. Lambert C, Dubuc JE, Montell E, Vergés J, Munaut C, Noël A and Henrotin Y: Gene expression pattern of cells from inflamed and normal areas of osteoarthritis synovial membrane. Arthritis Rheumatol 66: 960-968, 2014.

32. Collins CE, La DT, Yang HT, Massin F, Gibot S, Faure G and Stohl W: Elevated synovial expression of triggering receptor expressed on myeloid cells 1 in patients with septic arthritis or rheumatoid arthritis. Ann Rheum Dis 68: 1768-1774, 2009.

33. Tang J and Dong Q: Knockdown of TREM-1 suppresses IL- $1 \beta$-induced chondrocyte injury via inhibiting the NF- $\kappa \mathrm{B}$ pathway. Biochem Biophys Res Commun 482: 1240-1245, 2017. Attribution-NonCommercial-NoDerivatives 4.0 International (CC BY-NC-ND 4.0) License. 